A N N A L E S Annales de Bretagne et des Pays de l'Ouest

Anjou. Maine. Poitou-Charente. Touraine

109-4 | 2002

Les étrangers dans l'Ouest de la France (XVIII ${ }^{-}-X^{e}$ siècle)

\title{
Les Juifs étrangers en Maine-et-Loire pendant la Seconde Guerre mondiale
}

\section{Alain Jacobzone}

\section{(2) OpenEdition Journals}

Édition électronique

URL : http://journals.openedition.org/abpo/1530

DOI : $10.4000 / a b p o .1530$

ISBN : 978-2-7535-1488-1

ISSN : 2108-6443

\section{Éditeur}

Presses universitaires de Rennes

\section{Édition imprimée}

Date de publication : 20 décembre 2002

Pagination : 191-202

ISBN : 978-2-86847-794-1

ISSN : 0399-0826

\section{Référence électronique}

Alain Jacobzone, "Les Juifs étrangers en Maine-et-Loire pendant la Seconde Guerre mondiale », Annales de Bretagne et des Pays de l'Ouest [En ligne], 109-4 | 2002, mis en ligne le 20 décembre 2004, consulté le 02 mai 2019. URL : http://journals.openedition.org/abpo/1530; DOI : 10.4000/abpo.1530 


\title{
Les Juifs étrangers en Maine-et-Loire pendant la Seconde Guerre mondiale
}

\author{
Alain JACOBZONE \\ Professeur agrégé d'histoire, lycée Bergson - Angers
}

Encore mal connu, éclipsé une fois encore par le poids des événements survenus dans la capitale ${ }^{1}$, le calvaire des Juifs de province pendant la Seconde Guerre mondiale, étudié à travers l'exemple angevin, montre que la chasse dont ils furent l'objet fut systématique et d'une redoutable efficacité $^{2}$. La petite communauté réunie dans le Maine-et-Loire - environ 400 personnes en juin 1942 lorsque se préparent les premières rafles de juillet est très majoritairement composée de réfugiés arrivés depuis le début de la guerre, et le poids des Juifs indigènes, nés dans le département ou y résidant depuis longtemps est réduit : sur les 293 personnes inscrites sur l'état des Juifs de l'arrondissement d'Angers en octobre 1940, seules 29 sont nées dans le département et 67 s'y sont établies à des dates diverses, la plupart après les années 1920. C'est dire si le monde juif en Anjou, en dehors même de toute considération de nationalité, est étranger à la province, où il n'y a que peu de racines et donc une implantation et des relations forcément limitées et superficielles. C'est là une première constatation importante dans la perspective des épreuves à venir : elles seront affrontées le plus souvent dans l'isolement, sans le recours aisé à des appuis extérieurs que procurent des relations tissées de longue date et entretenues par des fréquentations prolongées. On sait bien que lorsqu'il s'agira, par la fuite et la plongée dans la clandestinité, de réagir face au danger qui menace, après l'instauration de l'étoile jaune en zone occupée en juin 1942, puis suite aux grandes rafles de juillet, les complicités seront essentielles, pour une cache, un transport, du ravitaillement ${ }^{3}$.

1. Pour un tableau du monde Juif en France à la veille de la guerre, voir KASPI, André, Les Juifs pendant l'occupation, Paris, Seuil, 1991, et PoznAnSki, Renée, Les Juifs en France pendant la Seconde Guerre mondiale, Paris, Hachette, 1994. On compte en France de 300000 à 330000 Juifs en 1939. Environ 100000 à 150000 résident à Paris et dans le département de la Seine. À Nantes on en compte 537 en octobre 1940, à Laval 97.

2. Pour un bilan global du génocide en Anjou : JACOBZONE, Alain, L'éradication tranquille, le destin des Juifs en Anjou (1940-1944), Vauchrétien, Ivan Davy éditeur, 2002.

3. CoHEn, Ascher, Persécutions et sauvetages, Juifs et Français sous l'occupation et sous Vichy, Paris, Les Éditions du Cerf, 1993. 
Les Juifs de nationalité étrangère constituent une partie importante de la population juive en Anjou. Dans l'arrondissement d'Angers en 1940, ils sont 46 soit $20 \%$; la plupart - les 2 tiers - sont originaires de l'Europe centrale et orientale, surtout de Pologne (16), mais aussi de Russie (7) et de Roumanie (5). Cette situation est à l'image de l'ensemble de la communauté juive de France, qui a vu le nombre des réfugiés d'Europe centrale fuyant les persécutions antisémites augmenter notablement depuis le début du siècle ${ }^{4}$. Si les Juifs français sont donc bien finalement les plus nombreux en 1940, il convient d'insister sur le fait que 20 \% d'entre eux sont nés à l'étranger et qu'ils ont fait l'objet de mesures de naturalisation dans les années trente ${ }^{5}$. Mais qu'ils soient français ou étrangers, ces Juifs nés en dehors du territoire national sont dans notre pays depuis longtemps puisque $82 \%$ y résident depuis plus de 10 années (33 d'entre eux sont même là depuis plus de 20 ans). Finalement, l'immigré de fraîche date, arrivé depuis la crise des années trente est, comme on pouvait s'y attendre un cas de figure assez rare $^{6}$ (9 individus au total sont arrivés après 1930). Bien sûr dans une même famille, surtout lorsqu'elle est nombreuse et que des enfants sont nés en France après l'arrivée des parents, les différences de statuts ne sont pas rares : ces derniers sont restés étrangers alors que leur progéniture est devenue française. C'est surtout au chef lieu que résident les Juifs étrangers : ils y sont deux fois plus nombreux que dans les communes rurales du reste de l'arrondissement; outre des capacités de logement plus importantes en ville, on peut penser qu'à l'exemple du précédent de la guerre 1914-1918, les autorités ont préféré diriger les étrangers vers la ville en estimant leur intégration plus facile que dans des milieux ruraux moins préparés psychologiquement à les recevoir ${ }^{7}$.

De 1940 à 1942, on observe quelques évolutions significatives ${ }^{8}$. Au niveau départemental cette fois, le poids des étrangers a considérablement augmenté. Ils sont, en proportion presque deux fois plus nombreux puisque leur part qui restait limitée à $20 \%$ dans l'arrondissement d'Angers bondit à 39 \% : ils sont 139 sur un total de 398 Juifs recensés en juin 1942 dans l'ensemble du département. La composition nationale, elle aussi a évolué et s'est diversifiée : si les Polonais avec 65 individus restent le groupe le plus nombreux et représentent avec les ressortissants des autres pays d'Europe centrale et orientale (Russes, Roumains, Tchécoslovaques, Hongrois et

4. Le livre noir de l'humanité, encyclopédie mondiale des génocides, Toulouse, Privat, 2001, p. 436-438.

5. JaCoBzone, Alain, L'éradication..., op. cit., p. 29.

6. Les restrictions ont été multipliées pour freiner l'immigration à partir des années 1930 : voir SCHOR, Ralph, L'opinion publique et les étrangers, Paris, Publications de la Sorbonne, 1985.

7. Jacobzone, Alain, En Anjou loin du front, Vauchrétien, Ivan Davy éditeur, 1988, p. 259-292.

8. Les documents produits par l'administration préfectorale sont d'une plus grande précision en 1942 : les bureaux ont perfectionné leurs méthodes et les Allemands, dans la perspective de la mise en place de l'étoile jaune puis des arrestations, exigent des renseignements rigoureux qui vont bientôt grandement leur faciliter la tâche. 
Yougoslaves) $56 \%$ du total des étrangers, on voit apparaître des représentants de l'Europe du nord et du sud (Belges, Néerlandais, Espagnols, 21 personnes) et les Allemands et ex-Autrichiens dont les effectifs atteignent 35 résidents; parmi ces derniers, une quinzaine sont présents dès le milieu des années 1930 dans une ferme qu'ils ont achetée et exploitent non loin de Saumur, à Chénehutte-les-Tuffeaux. On repère aussi 14 apatrides dont la situation au regard des règlements en vigueur n'a pu être éclaircie ou qui, faute de documents, n'ont pu faire la preuve de leur nationalité. Si la situation a ainsi évolué c'est en raison des mouvements intervenus entre les deux dates des recensements sur lesquels nous nous appuyons.

Des réfugiés sont repartis dès 1940, après l'armistice. Mais ces retours précoces ne concernent que rarement les étrangers. Pour un étranger ce sont quatre Français qui quittent l'Anjou. Cette disproportion a des origines légales et matérielles : l'État Français surveille les étrangers et limite une liberté de mouvement qui de toutes les façons est plus réduite pour des nouveaux arrivants installés depuis peu dans un pays où ils n'ont pas encore un réseau de relations développé. Dés le début donc la moindre mobilité des Juifs étrangers s'affirme et il s'agit là d'une première discrimination de fait et de droit qui aura - on le devine - de lourdes conséquences. Mais des nouveaux arrivants viennent aussi rejoindre les premiers installés. C'est le cas en particulier de 64 Juifs étrangers dits "réfugiés des côtes ", en fait chassés par les nazis des zones côtières considérées comme stratégiques par l'occupant et qui arrivent en 1941 dans le département où les autorités les répartissent dans 17 communes qui jusque-là n'abritaient pas de Juifs. Parmi eux, on compte justement une vingtaine d'Allemands et Autrichiens. Ce groupe particulier est digne d'intérêt à plus d'un titre. Il témoigne d'abord de la vision maléfique que les nazis ont des Juifs : s'ils les éloignent de la Loire-Inférieure et du Morbihan c'est qu'ils les imaginent capables de leur nuire gravement sur leurs arrières en cas de combats sur les côtes; d'autre part, arrivés tardivement, isolés dans des petites communes, mal intégrés, ils constituent un groupe particulièrement vulnérable et l'on comptera parmi eux un nombre considérable de victimes : $39 \%$ d'entre eux seront déportés dès les rafles de juillet 1942!

Autre groupe important de nouveaux arrivants, les travailleurs juifs étrangers qu'on installe au début de l'année 1942 dans un camp, à Clefs dans le Baugeois, pour des travaux de bûcheronnage destinés à fournir les mines de Lens ${ }^{9}$. Dès 1940, le gouvernement de Vichy prend des mesures pour regrouper et faire travailler sur des chantiers les étrangers et immigrés " en surnombre dans l'économie nationale ». Les Juifs étrangers, réduits au chômage par les mesures d'exclusion professionnelle qui visent les Juifs en général deviennent les principaux pensionnaires de ces camps. Au total, à Clefs, 150 personnes travailleront, les effectifs variant suivant les époques; la plupart, originaires de la région parisienne, sont étrangères (Polonais et Russes surtout), mais 17 Français (récemment naturalisés

9. Les effectifs du camp de Clefs ne sont pas inclus dans les comptages de juin 1942. 
ou même dénaturalisés?) y figureront. Le statut de ces travailleurs étrangers est particulier et le fonctionnement du camp - dont la gestion et la surveillance sont assurées par les autorités françaises - très différent de ce qu'on peut imaginer à la lumière de ce qu'on sait de l'univers concentrationnaire : les travailleurs sont, du moins théoriquement, volontaires et embauchés sur contrat de trois mois; ils peuvent donc renoncer et quitter le chantier; ils sont autorisés aussi à sortir du camp qui n'est pas clos, pour faire des achats personnels et ont même parfois, semble-t-il, la possibilité de faire venir une partie de leur famille. Ce fonctionnement plutôt libéral finit d'ailleurs par attirer l'attention des Allemands qui exigent bientôt un durcissement des conditions de détention...

Les enquêtes du début de l'été 1942 permettent de mieux appréhender quelques spécificités des Juifs étrangers au regard de leurs coreligionnaires français. La structure par âges en particulier est différente : les jeunes de moins de 15 ans et les vieux de plus de 60 ans sont, en proportion, deux fois moins nombreux chez les étrangers que chez les Français : $22 \%$ contre $41,5 \%$. Le monde des étrangers est donc avant tout un milieu d'adultes d'âge actif. Cela s'explique sans doute par leur histoire particulière : ces émigrants sont partis plutôt jeunes, dans la force de l'âge, alors que les anciens dans leur famille sont parfois restés sur place, rebutés par les difficultés du départ et préférant peut-être les risques réels mais connus d'un maintien sur place dans un pays où ils ont leurs racines, à la plongée dans l'inconnu. Sur place, devant affronter les difficultés de l'installation et de l'enracinement dans le contexte tendu des années trente, les nouveaux arrivants ont peut-être différé ou ralenti la fondation d'une famille en attendant... des jours meilleurs. Les Juifs étrangers ont donc autour d'eux moins d'enfants et surtout moins de vieillards que les Français qui ont fait souche depuis plus longtemps. Cette particularité démographique n'est pas sans importance pour la suite des événements, car aux moments décisifs les Juifs étrangers pourront peut-être plus facilement mettre en branle des groupes plus réduits et moins lourdement chargés d'enfants ou de vieillards. D'un autre côté, ils auront d'autres handicaps qui leur sont plus spécifiques, en particulier une moins complète intégration, un réseau de relations plus réduit et une moins bonne connaissance du pays dans son ensemble.

Autre différence, surprenante : si le chômage touche fortement le monde juif angevin dans son ensemble ${ }^{10}$, il est, au rebours de ce qu'on peut attendre, plus modéré parmi les étrangers : alors qu'en juin 1942, 93 \% des Juifs français se déclarent sans profession, seulement $74 \%$ des étrangers se disent dans la même situation. Établis depuis moins longtemps en France,

10. Le chômage parmi les Juifs est énorme : plus encore que le résultat des conditions de la guerre, il s'agit d'une conséquence dramatique des mesures d'exclusions professionnelles et de l'aryanisation des biens juifs qui privent ces derniers de tout moyen de travailler et les réduit souvent à survivre des aides publiques, ce qu'on ne manquera pas bien entendu de leur reprocher. Sur ces mesures et leur application rapide et sans faiblesse dans le Maine-et-Loire, voir JACOBZONE, Alain, L'éradication..., op. cit., p. 62-87. 
n'y ayant pas encore atteint le stade de la réussite par l'acquisition de l'atelier ou de l'échoppe, ils sont encore très majoritairement ouvriers : les mesures d'aryanisation des biens juifs prises par les nazis et Vichy les touchent donc moins sans doute que les Français et, comme simples ouvriers ils parviennent peut-être un peu plus facilement à trouver du travail.

Ce monde des Juifs étrangers avant les rafles étant un peu mieux cerné, tentons maintenant d'évaluer le tribut qu'il a dû payer à la folie antisémite déchaînée par les nazis à partir de juillet 1942 en Anjou. Ce sont bien, et de loin, les étrangers qui furent les victimes les plus nombreuses du génocide angevin, à la fois en valeur absolue et en part relative. Si l'on met de côté pour le moment le cas des Juifs de Clefs, 119 Juifs étrangers ont été déportés ce qui représente $73 \%$ des déclarés de juin contre seulement (!) $45 \%$ des Français. Ce pourcentage déjà énorme, bondit à 87,5 \% si l'on considère qu'au moment où se produisent les rafles, 3 Juifs étrangers sont décédés et 22 autres ont pris la fuite et quitté l'Anjou (il s'agit là d'un chiffre minimum). On ne doit pas s'étonner de cette différence au détriment des étrangers : en 1942, lorsque se décident et s'organisent les rafles, les nazis obtiennent la collaboration des autorités administratives et policières de Vichy en zone libre en concédant une certaine autonomie aux autorités françaises et en promettant que seuls les Juifs étrangers seront arrêtés ${ }^{11}$. On a donc, en haut lieu, froidement décidé de faire porter le poids des opérations essentiellement sur ces populations étrangères et il n'est donc pas étonnant qu'elles en aient fait les frais : sur ce point l'exemple angevin n'a rien de très spécifique. Encore faut-il opérer quelques distinctions à l'intérieur même du groupe des Juifs étrangers. Les Juifs Allemands et exAutrichiens ont été les plus durement touchés : ils étaient 34 et 33 ont subi la déportation. Les nazis sont sans doute particulièrement attachés à leur perte car, finalement ils ne peuvent admettre que des Juifs soient Allemands et ils sont persuadés que, d'une certaine manière proches d'eux, ils sont particulièrement dangereux. Ce sont des réfugiés politiques souvent opposants au nazisme. Ajoutons qu'ils sont immigrés de fraîche date, et ainsi plutôt mal intégrés et qu'une quinzaine d'entre eux étaient concentrés dans la ferme modèle de Chénehutte-les-Tuffaux, donc faciles à saisir. Ajoutons que pour les autorités françaises ces individus ont le double défaut d'être Juifs et Allemands et qu'à ce dernier titre on n'est pas tenu de les ménager à la différence de l'occupant. Pourtant, les pertes chez les Roumains sont tout aussi lourdes : 12 sur 13 furent déportés. Les apatrides, avec $85 \%$ de déportés ont aussi beaucoup souffert : par définition inclassables, sans la protection d'aucun statut national, ils sont une proie sans défense. Puis viennent les Polonais, un peu moins touchés avec $68 \%$ de victimes. Relativement nombreux, parfois depuis longtemps en France, ils ont peut-

11. Ces négociations ont été reconstituées en détail dans un ouvrage capital par KLARSFELD, Serge, Vichy-Auschwitz, Paris, Fayard, 1993. Avant lui deux historiens avaient déjà montré la lourde implication des autorités françaises de Vichy dans la préparation de la déportation : Marrus, Michaël et PAXTON, Robert O., Vichy et les Juifs, Paris, Calmann-Lévy, 1981. 
être pu un peu mieux s'organiser et relativement mieux se protéger. Les ressortissants d'autres pays sont nettement moins nombreux ce qui rend aléatoire l'étude statistique. Signalons pour terminer le cas intéressant des Juifs ressortissant des pays alliés ou neutres. Bien que pour des raisons tactiques et diplomatiques, les autorités nazies aient décidé de les ménager au moins jusqu'en 1943, dans le Maine-et-Loire ils n'ont guère été épargnés dès 1942 : 4 des 5 Suisses et les 5 Turcs enregistrés ont été arrêtés. Par contre les 2 Juifs espagnols que comptait le département ont été vigoureusement défendus par leur ambassade et ont pu le quitter sans encombre.

La géographie de la déportation est éclairante : avec $62 \%$ de victimes, les Juifs étrangers d'Angers ont été moins touchés que leur équivalent à la campagne où $85 \%$ d'entre eux ont été arrêtés. Le rapport est inverse dans le cas des Juifs français. Sans doute était-il plus facile à un Juif étranger de se cacher en ville et d'y trouver des complicités. Dans les petites communes on avait au contraire envoyé des familles entières, faciles à surveiller et à enlever. Enfin la discrimination a joué à plein contre les étrangers dans le cas où une intervention ou une procédure ont pu aboutir à une libération après les rafles. Ces cas ne sont pas négligeables puisque 32 personnes figurant sur les listes de juin ont été arrêtées mais ne figurent plus sur les listes de déportés ce qui semble indiquer une libération in extremis. Or, seules 2 d'entre elles sont étrangères. On sait bien que dans certains cas - rares - la préfecture intervient, surtout lorsqu'elle apprend après les rafles et après une virulente protestation écrite des autorités juives de Paris, que de nombreux Juifs français ont été arrêtés en violation des accords passés. Mais, justement, les autorités françaises ne réagissent qu'en faveur de personnalités françaises dont le cas leur paraît digne d'intérêt : des conjoints de non juifs, catholiques avérés de surcroît, des épouses de prisonniers de guerre français, des anciens combattants, des personnalités connues dans le département. Par contre on ne possède aucune trace d'intervention en faveur d'étrangers...

Ceci nous conduit à nous interroger sur les causes du calvaire particulier vécu en Anjou par les Juifs étrangers. De quoi, finalement ont-ils été victimes? L'hypothèse qui vient si naturellement à l'esprit qu'on en négligerait presque de poser la question est que, tout simplement, l'antisémitisme nazi a fait des ravages. Le fait n'est pas niable et les preuves sont abondantes : on peut facilement démontrer que les nazis présents à Angers sont des fanatiques et que de surcroît ils ont mis un zèle particulier à assouvir leur passion et à appliquer les consignes de leur hiérarchie. Ils sont mêmes allés au-delà de ces instructions, les outrepassant pour arrêter des Juifs qui n'auraient pas dû l'être C'est ainsi que dès juillet 1942, ils raflent un nombre important de Juifs français prétextant sans en fournir la preuve qu'ils n'ont pas respecté les règlements en vigueur les visant. Ils ne tiennent pas compte non plus des restrictions d'âge qui théoriquement avaient été mises aux arrestations en juillet. Dès le fameux convoi $\mathrm{n}^{\circ} 8$ qui quitte Angers le 20 juillet 1942, 50 Juifs français sont enlevés et au total 99 ont été 
déportés jusqu'en 1944 ce qui représente $45 \%$ des inscrits de la liste de juin (et 61 \% de ceux qui sont réellement présents au moment des rafles entre 1942 et 1944). Et si l'on établit pour le Maine-et-Loire un état final de la déportation, force est de constater qu'il est particulièrement lourd, plus que le bilan moyen français ${ }^{12}$. C'est justement parce, dans ce département les Juifs français ont été peu ou prou aussi durement traités que leurs compagnons d'infortune étrangers. Et si partout ailleurs on avait procédé à l'identique, le bilan français aurait approché, en proportion, les hécatombes enregistrées à l'est en Pologne, ou dans les pays Baltes. Incontestablement, le Kommandeur SS Hauptsturmfürher Ersnt qui dirige l'antenne Sipo SD d'Angers, a fait du zèle avec son adjoint Schulz Haike à la tête de la section IV B de la Gestapo, plus particulièrement chargée de la question juive. Il a réussi finalement à faire partir le seul des 6 convois qui avaient été planifiés au départ de la province quelques semaines auparavant. Et justement, il y parvient en y adjoignant les Juifs français qui normalement auraient dû en être exclus. Sans doute les nazis se sentent-ils en force à Angers puisque la ville est une capitale de l'administration allemande qui a la haute main sur 17 départements français s'étendant des Pyrénées à la pointe de la Bretagne. On peut estimer à 6000 le nombre des Allemands occupant la ville ${ }^{13}$ !

Pourtant l'explication de la dureté du sort fait aux Juifs en général et aux étrangers en particulier par le fanatisme et le zèle antisémites des nazis nous paraît insuffisante car elle ne rend pas compte de l'action et de la motivation des auxiliaires français de la chasse aux Juifs. Aucun doute n'est possible : l'administration angevine de l'État français a prêté sans barguigner son concours à l'occupant dans ses opérations anti-juives : des mesures discriminatoires de 1940 - dont on sait bien d'ailleurs qu'elles sont souvent d'initiative française - à l'étoile jaune en passant par l'aryanisation des biens juifs, la préfecture et ses services sont très actifs sous l'impulsion du préfet départemental puis régional Roussillon ${ }^{14}$. Au moment des rafles du 15 au 17 juillet, puis au départ du convoi $\mathrm{n}^{\circ} 8$ le 20, ce ne sont pas moins que 16 à 29 policiers français qui prêtent leur concours aux arrestations ou au convoiement des Juifs des départements limitrophes qui sont

12. Au total, avec les travailleurs du camp de Clefs, il y a eu au moins 319 déportés en Maine-et-Loire et 324 victimes en ajoutant 2 suicidés, un fusillé, un fuyard abattu et une personne assommée en gare d'Angers. Calculée par rapport aux seuls présents sûrs en Juillet de la liste de juin 1942, on arrive à une proportion moyenne de déportés de $73 \%$; cette proportion peut même monter à $88 \%$ si l'on considère que nous ne sommes pas renseignés sur le cas d'un certain nombre d'inscrits de juin 1942, mais qui ont sans doute fui. Après la dernière rafle de janvier, il reste 22 Juifs présents. Ces statistiques nous font penser que la déportation en Anjou a été plus systématique et totale que dans beaucoup d'autres endroits en France. Rappelons que l'estimation moyenne de la déportation en France, calculée par rapport aux présents de 1939, tourne autour de $25 \%$.

13. LAVAUD, Édouard, Angers occupée... Les formes de l'occupation Allemande, mémoire de maîtrise, Université d'Angers, 1998.

14. Les préfets de Maine-et-Loire, sous la direction de Jean-Luc MARAIS, avec la collaboration de Céline Lambert, Presses Universitaires de Rennes, 2000. 
dirigés vers le grand séminaire de la capitale régionale, rue Barra, puis le jour du départ vers le quai militaire de la gare du Maroc. En septembre 1942, le préfet n'hésite pas à écrire que si ses rapports sont difficiles avec l'administration allemande en général, par contre ils sont excellents... avec les SS! Le mois suivant après la deuxième vague de rafles, il est fier d'annoncer au ministre de l'Intérieur que

"malgré les difficultés de toutes sortes j'ai pu néanmoins assurer l'exécution de ces instructions [celles données par les SS] dans les meilleures conditions possibles sans qu'aucun accident vienne à se produire. Une escorte de 22 gendarmes français a assuré le transfert jusqu'au camp de concentration de Drancy. "

Mais pourquoi cette attitude? Outre que le préfet Roussillon est parfaitement au courant et partage - les documents le prouvent - le calcul de Bousquet et de Laval qui pensent que pour obtenir des Allemands qu'ils accordent une autonomie de décision à la France en matière de police, il convient de leur donner des gages, en particulier sur la question juive, il est sans doute mû par une franche xénophobie d'ailleurs largement partagée. En juin 1941, l'administration est saisie par une demande en révision d'une mesure de dénaturalisation d'un médecin juif français d'origine roumaine exerçant à l'école de cavalerie de Saumur. L'instruction établit que ce médecin est très apprécié, que son comportement est irréprochable et que rien ne justifie finalement la mesure dont il est victime; le sous-préfet de Saumur après lecture du rapport de police doit conclure que rien ne s'oppose à ce que la mesure de dénaturalisation soit rapportée, mais ajoute que, malgré tout, il n'y est pas favorable. Et, en deux mots il explique pourquoi : "France d'abord! »; dans la marge le préfet ajoute : « Je suis bien d'accord. " Tout est dit et ce médecin sera parmi les premières victimes des arrestations de juillet : on mesure à travers ce simple épisode la mesure et la portée de l'hostilité aux étrangers de deux hauts responsables de l'administration angevine. Par contre on cherchera vainement dans leurs déclarations publiques ou privées des traces d'un antisémitisme pur : les Juifs, même s'ils sont français sont considérés avant tout comme des étrangers, en marge de la communauté nationale. De manière très significative, toutes les affaires juives - même lorsqu'il s'agit de Juifs français - sont gérées dans les bureaux de la préfecture par les services des étrangers. L'optique de cette gestion est parfois tout simplement comptable : en 1941 à la demande de Vichy l'administration angevine recherche les étrangers " en surnombre dans l'économie nationale ». De manière très significative l'enquête aboutit à l'établissement d'une liste d'étrangers qui ont la particularité d'être tous Juifs! Suit cette explication :

" [...] Bon nombre d'israélites étrangers expulsés du Morbihan et de la Loire-Inférieure par les autorités d'occupation, ont été répartis dans le département et sont hébergés par le service des réfugiés; ces étrangers qui sont indigents et qui ne peuvent se livrer à aucun travail continu faute d'employeurs et d'autorisation des services de la main-d'œuvre, sont une charge pour la collectivité ( $12 \mathrm{~F}$ par jour en moyenne) et sont de ce fait en surnombre dans l'économie nationale. " 
Mais ce rapport est suivi d'une suggestion dont les conséquences échappent - on peut l'espérer - au fonctionnaire du bureau des étrangers :

"Leur séjour dans un camp de concentration serait moins onéreux que l'hébergement individuel. J'ai en conséquence l'honneur de proposer à Monsieur le préfet l'internement dans un camp de concentration des étrangers israélites figurant sur la liste ci-jointe..."

Le représentant de Vichy donne suite et, en son nom, le secrétaire général signe, le 18 septembre 1941, un arrêté d'internement au camp de Baunela-Rolande (Loiret) visant 8 personnes, dont 6 Polonais, tous des hommes. Lorsque quelques mois plus tard, les Allemands cherchent des victimes pour remplir leurs premiers convois dirigés vers Auschwitz, ils les trouvent à disposition dans ce camp! Le 28 juin 1942 s'ébranle le convoi n ${ }^{\circ} 5$ emportant 5 de ces juifs ramassés en Maine-et-Loire : ce sont les premières victimes de la déportation dans ce département avant même les 136 qu'emportera le convoi $n^{\circ} 8$ moins d'un mois plus tard...

N'oublions pas le contexte : la défaite de 1940 pose la question des responsabilités : et une des réponses apportées par Vichy est l'insuffisance de l'esprit national affaibli par la présence de trop nombreux étrangers. La renaissance de la France passe donc par une véritable purge qui va consister d'abord à les mettre à l'écart des centres de décision puis de la société tout entière. Cette politique bien sûr explique que Vichy ait d'abord voulu livrer les Juifs étrangers et fait le jeu des nazis qui apparaissent ainsi comme d'utiles auxiliaires de la Révolution Nationale. Loin d'être propre aux responsables de la préfecture cette réaction xénophobe semble partagée par une grande partie de l'opinion publique. Le premier rapport la concernant, signé par le secrétaire général le 24 août 1940, signale que

"l'opinion loue, d'une manière générale, les mesures de tous ordres prises par le gouvernement pour assurer le redressement matériel et moral du pays. Elle a accueilli avec faveur le rétablissement de l'autorité, de la discipline, les mesures prises contre les étrangers."

La presse affirme des positions identiques : Le Petit Courrier, journal modéré, réputé en phase avec l'opinion majoritaire en Anjou, commente ainsi le 19 octobre 1940 la publication du premier statut des Juifs qui inaugure la longue série des discriminations qu'ils vont subir :

«Le gouvernement dans son œuvre de reconstruction nationale, a dû, dès les premiers jours, étudier le problème de Juifs et des étrangers, qui ayant abusé de notre hospitalité, n'ont pas peu contribué à notre défaite. Partout, et spécialement dans les services publics, l'influence des Juifs se fait sentir, insinuante et finalement, décomposante [...]. Notre gouvernement entend, dans une absolue sérénité, faire œuvre de représailles. Il respecte les personnes et les biens des Juifs. Il les empêche d'exercer certaines fonctions [...]. Notre désastre nous impose de regrouper les vrais Français. Il ne s'agit pas d'une persécution mais d'une indispensable sécurité. "

Remarquons au passage l'allusion aux "vrais Français " : elle sousentend qu'un Juif français doit en fait être considéré comme un étranger, 
il reste un étranger, inassimilable. Il semble bien que la crise puis la montée des périls à la fin des années trente, la guerre enfin aient bien généré dans cette province ou pourtant ils n'étaient ni nombreux ni mal considérés ${ }^{15}$, des réactions de méfiance vis-à-vis des étrangers en général, surtout s'ils sont Juifs : c'est cette évolution que suggère clairement un rapport de police concernant les Juifs allemands réfugiés dans une ferme qu'ils exploitent dans le Saumurois :

« Depuis 1934 ces étrangers ont travaillé dans leur exploitation sans avoir beaucoup de rapports avec la population de la région et sans donner lieu à des remarques désagréables quant à leur conduite privée. Mais ils ont donné lieu à de nombreuses dénonciations pour espionnage, tant de l'autorité militaire française que de celle des civils."

On pourrait aussi évoquer l'évolution des attitudes vis-à-vis des réfugiés espagnols, d'abord bien accueillis puis considérés comme une lourde charge dont on voudrait bien se débarrasser : le sous-préfet de Cholet prévient en octobre 1939 :

" Je tiens à vous signaler que la présence en France des réfugiés espagnols, hébergés et nourris aux frais de l'État (qui à l'heure actuelle a des charges plus impérieuses), est assez sévèrement appréciée par la population et par les maires de mon arrondissement qui ne cessent de me demander ce qu'on attend pour les renvoyer chez eux."

La presse se fait largement l'écho de ces réactions xénophobes dès l'avant guerre : elle se scandalise qu'un Juif étranger, en assassinant un diplomate de l'ambassade d'Allemagne à Paris en 1938, vienne compliquer nos relations avec ce pays ${ }^{16}$. Citons par ailleurs ce commentaire du Petit Courrier à l'annonce de l'ouverture par le gouvernement de la République des camps de travail pour étrangers en novembre 1938 :

«Ma foi, voilà qui est tout à fait bien et nous y applaudissons des deux mains! Vous avouerez en effet que nous avons assez à faire avec nos chenapans à nous sans être encore empoisonnés par tous ceux dont les pays voisins se sont débarrassés à juste titre et sont venus se réfugier en France. Nous en avons assez de voir les ouvriers français d'une entreprise vouloir continuer ou reprendre le travail et en être empêchés par quelque Schwartzeinkorpf ou autre Chahutsky, parfaitement irresponsable et insaisissable sous aucune forme, et qui avaient réussi à la terroriser. "

Les Juifs ont donc été fondamentalement et généralement considérés comme des étrangers même lorsqu'ils possédaient la nationalité française. Ils sont perçus comme étant, par définition, en marge, à part. C'est ce qui explique sans doute que, jusqu'aux rafles qui sont jugées excessives et cho-

15. JeAnneAu, Jacques, "Les étrangers en Maine-et-Loire dans les années trente ", Mémoires de l'Académie d'Angers, tome X, 1987-1988 et RosETINY, Dominique, Les étrangers dans le Maine-et-Loire entre 1880 et 1939, mémoire de maîtrise, Université d'Angers, 2000.

16. Cet événement est le prétexte de la nuit de cristal, première grande vague de violence meurtrière dirigée contre les Juifs en Allemagne (novembre 1938). 
quantes, non dans leur principe mais dans leur forme ${ }^{17}$, l'opinion de la province ne réagit absolument pas aux mesures d'exclusion et de marginalisation des Juifs. Elles n'apparaissent peut-être que comme la sanction de droit d'un état de fait généralement admis. Une affaire qui a agité en 1942 un petit village du Sud-Saumurois montre bien à quelles conditions, un Juif Français peut être considéré comme quasiment assimilé à la communauté locale et nationale. Après l'arrestation et la déportation de ses propriétaires parisiens une petite maison a été occupée par un jeune homme sur le point de se marier. Les gendarmes décrivent ainsi les réactions de la population : "La population du Coudray a été émue de la réaction de L... : si le fait pour R... d'être Juif est un préjugé défavorable, il n'empêche qu'il est Français, ancien combattant et qu'il a un fils prisonnier de guerre. " C'est bien une manière de dire qu'il y a des Juifs acceptables et d'autres qui le sont moins. Mais pour l'être que de conditions n'exige-t-on pas : le Juif spolié du Coudray avait tous les sacrements qui le lavaient presque de ses origines raciales. Français certes, né à Paris, avocat dans la capitale, ancien combattant et père de prisonnier de guerre, chevalier de la Légion d'honneur, de surcroît exploitant quelques arpents de vigne comme tout le monde dans ce pays à vin...

À l'aune de ce qu'on exigeait alors d'un Juif Français pour n'être pas traité à part, il est peu de " vrais Français " de vieille souche qui eussent pu satisfaire à des telles exigences. Alors les étrangers...

17 Sur les réactions aux rafles en Anjou : JACOBZONE, Alain, L'éradication..., op. cit., p. 107115 et $127-128$. 


\section{RESUME}

Les Juifs forment une toute petite communauté en Anjou pendant la Seconde Guerre mondiale. Parmi eux, les étrangers, surtout originaires de l'Europe centrale et orientale, constituent une importante minorité. La plupart se sont réfugiés dans le Maine-et-Loire depuis le début de la guerre.

Les Juifs étrangers seront pour la plupart déportés. Mais dans le Maineet-Loire les Juifs français sont presque aussi touchés. Fondamentalement, tous les Juifs même français, sont considérés comme des étrangers. L'administration française et l'opinion locale paraissent convaincus qu'ils sont trop nombreux et que leur influence est néfaste. Cette attitude fait le jeu des nazis et favorise leurs entreprises meurtrières.

\section{ABSTRACT}

The Jews formed a very small community in Anjou during the Second World War. Among them, foreigners especially from central and eastearn Europe constituted a large minority. Most of these had taken refuge in Maine-et-Loire during the begening of the war.

A large majority of the foreign Jews were to be deported but French Jews in Maine-et-Loire were almost as badly hit. Basically, all Jews, even French ones, were regarded as foreigners The french authorities and local people seemed convinced that there were too many of them and that they had a bad influence. That attitude of course suited the nazies and facilitated their murderous ambitions. 